\title{
IN VIVO AND IN VITRO EVALUATION OF EFFICACY OF HUMIC ACID AGAINST AFLATOXINS
}

\author{
Yasser M. Abd El-Shafea, Gihan M. Hammoud, Nivin S. Nail, Asmaa A. Salem and \\ Olaa A. Wahdan* \\ * Regional Center for Food and Feed (RCFF), Agricultural Research Center (ARC), Giza, \\ Egypt.
}

\begin{abstract}
Aflatoxins (AFs), a group of closely related, extremely toxic mycotoxins produced by Aspergillus flavus and A. parasiticus, can occur as natural contaminants of food and feeds. AFs have been shown to be hepatotoxic, carcinogenic, mutagenic and teratogenic to different animal species. Therefore reducing their toxicity in vivo is of major interest. The potential of humic acid (HA) was evaluated for affinity to adsorb AFs from aqueous solution in vitro and for reducing the AFs-induced toxicity and oxidative stress in rat. In vitro study, three concentrations of HA (20, 60, and $100 \mathrm{mg} / \mathrm{ml}$ aqueous solution) and three concentrations of AFs (20,60 and 100ppb) at three interaction time (1, 2 and $3 \mathrm{hr})$ were tested. The results indicated that HA had a high capacity of adsorbing AFs at different tested concentrations and adsorption not significantly affected by increasing interaction time. The adsorption affinity was increased by elevation HA concentration and decreasing AFs concentration. The in vivo result indicated that rats administrated orally with 1 and $2 \mathrm{mg}$ AFs/ $\mathrm{kg}$ body weight (b.w)/once/week for 6 week showed significant dose and time- dependant increase in serum alanine transaminase (ALT), aspartate aminotransferase (AST), alkaline phosphatase (ALP), gamma glutamyl transpeptidase (GGT), creatinine, urea, triglycerides, total cholesterol and lipid peroxidation product (malondialdehyde, MDA) and significant time and dose- dependent reduction of serum albumin and total protein (TP), plasma reduced glutathione (GSH), glutathione -S- transferase (GST), superoxide dismutase (SOD) contents. Hyperglycemia was observed in AFs ingested rats in time and dose dependant manner. While, HA intake at two examined doses (200 and 400mg/kg b.w/daily) did not alter any of measured parameters along experimental periods except plasma GSH which enhanced significantly along experimental periods. The combined treatment showed significant improvement in all tested parameters. This improvement was more pronounced in the groups received the high dose of HA especially low AFs- co treated group. It could be concluded that HA has a potential activity and protective effect against AFs toxicity and this protection was dose dependant.
\end{abstract}

Key words: Aflatoxins, Humic acid, Binding capacity, Oxidative stress and Biochemical parameters.

\section{INTRODUCTION}

Aflatoxins (AFs) are secondary toxic fungal metabolites produced by Aspergillus flavus and A. parasiticus. There are four naturally occurring AFs, the most hepatotoxic being aflatoxin $\mathrm{B}_{1}\left(\mathrm{AFB}_{1}\right)$, and three structurally similar compounds namely aflatoxin $\mathrm{B}_{2}\left(\mathrm{AFB}_{2}\right)$, aflatoxin $\mathrm{G}_{1}\left(\mathrm{AFG}_{1}\right)$ and aflatoxin $\mathrm{G}_{2}\left(\mathrm{AFG}_{2}\right)$. AFs not only contaminate our food stuffs but are also found in edible tissues, milk and eggs after consumption of contaminated feed by farm animals (Saini and Kaur, 2012; Bennett and Klich, 2003 and Fink-Gremmels, 1999). AFs have been detected in cereal grains, whole wheat and rye breads, oil seeds, fermented beverages made from grains, milk, cheese, meat, nut products, fruit juice and 
numerous other agricultural commodities (Bullerman, 1986), therefore the presence of AFs or toxigenic fungi in foods presents a potential hazard to human and/or animal health.

AFs are well known for their hepatotoxic and hepatocarcinogenic effects (Wogan, 1999) and as potent mutagens, carcinogenic, teratogenic, and immunosuppressive and also inhibit several metabolic systems, causing liver, kidney and heart damage. These toxins have been incriminated as the cause of high mortality in livestock and some cases of death in human beings (Wangikar et al., 2005). The diseases caused by AFs consumption are loosely called aflatoxicoses. Acute flatoxicosis results in death; chronic aflatoxicosis results in cancer, immune suppression, and other "slow" pathological conditions with increased susceptibility to infectious diseases and growth retardation in young individuals (Kensler $\boldsymbol{e t}$ al., 2011 and Hsieh, 1988).

The mechanism of $\mathrm{AFB}_{1}$ toxic effect has been extensively studied. It has been shown that $\mathrm{AFB}_{1}$ is activated by hepatic cytochrome $P 450$ enzyme system to produce a highly reactive intermediate, $\mathrm{AFB}_{1}-8,9$-epoxide, which subsequently binds to nucleophilic sites in DNA, and the major adduct 8,9-dihydro- 8 -( $N^{7}$ guanyl $)-9$-hydroxy- $\mathrm{AFB}_{1}\left(\mathrm{AFB}_{1} N^{7}\right.$-Gua) is formed. The formation of $\mathrm{AFB}_{1}$-DNA adducts is regarded as a critical step in the initiation of $\mathrm{AFB}_{1}$-induced carcinogenesis (Sharma and Farmer, 2004; Klein, 2002; Preston and Williams, 2005). Although the mechanism underlying the toxicity of AFs is not fully understood, several reports suggest that toxicity may ensue through the generation of intracellular reactive oxygen species (ROS) like superoxide anion, hydroxyl radical and hydrogen peroxide $\left(\mathrm{H}_{2} \mathrm{O}_{2}\right)$ during the metabolic processing of $\mathrm{AFB}_{1}$ by cytochrome $P 450$ in the liver. These species may attack soluble cell compounds as well as membranes, eventually leading to the impairment of cell functioning and cytolysis (Towner et al., 2003; Sohn $\boldsymbol{e t}$ al., 2003; Berg et al., 2004). Biological compounds with antioxidant properties contribute to the protection of cells and tissues against deleterious effects of ROS and other free radicals.

Consequently, large-scale, practical, and cost-effective methods for detoxifying aflatoxin (AF)-contaminated feedstuffs currently are in great demand.

A number of methods, including physical, chemical and biological techniques have been used to protect animals from the toxic effects of AFs, but most of these methods are costly, time-consuming, and only partially effective (Doyle et al., 1982 and Phillips $\boldsymbol{e t}$ al., 1990). One of the most practical approaches is the use of nonnutritive adsorbents, which bind the mycotoxins and inhibit their adsorption from the gastrointestinal tract (Ramos $\boldsymbol{e t}$ al., 1996), but not all adsorbents are equally effective and several adsorbents have been shown to impair nutrients utilization (Kubena et al., 1993 and Scheideler, 1993). According to Ramos and Hernandez (1996), as an ideal adsorbent, it should have a high affinity to $\mathrm{AF}$, resulting in the formation of a strong complex with little risk of dissociation. It also should have a high binding capacity to prevent saturation.

Moreover, natural substances that can prevent $\mathrm{AFB}_{1}$ toxicity would be helpful to human and animal health with minimal cost in foods and feed (Kumar et al., 2007).

Humic acid (HA) is a group of high-molecular weight polymers that result from decomposition of organic matter, particularly dead plants. It exist abundantly in peat, soil, well water, and other sources (Hartenstein, 1981). It consists of a mixture of closely related complex aromatic polymers, the exact composition of which varies with HA from different geographic locations. Chemical and infrared spectroscopic analyses revealed the presence of aromatic rings, phenolic hydroxyl, ketone carbonyl, quinone carbonyl, carboxyl, and alkoxyl groups in HA (Stevenson, 1985). 
Therapeutic and medicinal use of humic substances was already known in the Babylonian times, and during the Roman Empire mud baths were used to treat a number of ailments (Priegnitz, 1986). HA were also used as folk remedies for a wide variety of ailments (Lotosh, 1991). HA is the most explored group of humic substances. HA has been used as an antidiarrheal, analgesic, immunostimulatory, and antimicrobial agent in veterinary practices in Europe (Rath $\boldsymbol{e t}$ al., 2006). Several interesting and potentially clinically important biological activities were recently associated with various types of HA, including antiviral properties (Klocking $\boldsymbol{e t} \boldsymbol{a l}$., 1983) and proliferation of lymphocytes (Ioone $\boldsymbol{e t}$ al., 2003). Also, addition of HA into the feed of cultured animals results in improved growth and health (Islam et al., 2005).

HA are ubiquitous and are found wherever matter is being decomposed or has been transposed, as in the case of sediments. Humic substances have demonstrated a strong affinity to bind various substances, such as heavy metals (Madronova' et al., 2001), herbicides (Ne'gre et al., 2001), different mutagens (Sato et al., 1987 and Cozzi et al., 1993), monoaromatic (Nanny and Maza, 2001) and polycyclic aromatic compounds (Kollist-Siigur et al., 2001), minerals (Elfarissi and Pefferkorn, 2000), and Bacillus subtilis bacteria (Fein et al., 1999).

A recent report by Van Rensburg et al. (2006) and Ghahri et al. (2010) has described that HA could alleviate some of the toxic effects of AF in growing broilers.

Our major objectives in this study were to evaluate the ability of HA to bind AFs from aqueous solution and to evaluate the safety and the protective effects of HA against AFs- induced toxicity and oxidative stress in rats.

\section{MATERIALS AND METHODS}

\section{Chemicals}

Humic acid were obtained from Loba chemie (Wodehouse Road, Mumbai) and all other chemicals and solvents were of analytical grade and purchased from Merck (Darmstadt, Germany), unless stated otherwise. Kits used for the estimation of GSH, GST, SOD and MDA were purchased from Biodiagnostic, France.

\section{Microorganism}

Aspergillus flavus NRRL (3145) was obtained from the National Research Center (Dokki, Giza).

\section{Standard Aflatoxins}

Aflatoxins $B_{1}, B_{2}, G_{1}$ and $G_{2}$ were obtained from Sigma Chemical Company (St. Louis, Mo USA).The purity of these materials were checked by thin layer chromatography [silica gel aluminium sheets 20x20 cm purchased from Merck (Darmstadt, Germany) using a mixture of diethyl ether: methanol: distilled water (96: 3: 1, v/v/v) as a running solution] and each gave one spot.

\section{Aflatoxins production and assays}

AFs were produced by the method of Davis et al. (1966) using liquid medium, yeast extract sucrose (YES) as substrate. Culture filtrates were extracted with chloroform $(1: 2, \mathrm{v} / \mathrm{v})$ (Abd El-Mageed, 1987). The chloroform extract was evaporated until obtaining dry film using rotary evaporator. Then the obtained dry film containing AFs was divided into 2 parts, $1^{\text {st }}$ part was reconstituted with hydroalcoholic solution (methanol/water $1: 1, \mathrm{v} / \mathrm{v}$ ) and used for in vitro study while the other part was reconstituted with dimethyl sulfoxide (DMSO) and used for in vivo study. 


\section{Determination of aflatoxins concentration in solution}

The concentration of AFs in DMSO or hydroalcoholic solution was determined using HPLC technique (Agilent 1100 Series U.S.A with column $C_{18}$, Lichrospher 100 RP-18, $5 \mu \mathrm{m} \times 25 \mathrm{~cm})$ as follows: The mobile phase consisted of water: methanol: acetonitrile $(54: 29: 17, \mathrm{v} / \mathrm{v} / \mathrm{v})$ at a flow rate of $1 \mathrm{ml} / \mathrm{min}$. The excitation and emission wavelengths for all AFs were 362 and 460nm (Flourcenses detector), respectively (Roos et al., 1997).

\section{Ability of humic acid to adsorb aflatoxins in vitro}

The hydroalcoholic containing AFs solution consisted of a mixture of $\mathrm{AFB}_{1}, \mathrm{AFB}_{2}$, $\mathrm{AFG}_{1}$ and $\mathrm{AFG}_{2}$ at a total concentration of $12684 \mathrm{ppb}$ in the ratio of 8: 3:2: 1, respectively used to prepare 3 final concentrations of AFs (20,60 and $100 \mathrm{ppb})$.

Three concentrations of HA $(20,60$ and $100 \mathrm{mg} / \mathrm{ml})$ were individually weighed into glass tubes (three replicates per sample) and the amount of AFs (20, 60 and $100 \mathrm{ppb}$ ) in hydroalcoholic solution were separately added. After a reaction time of $1 \mathrm{hr}, 2 \mathrm{hr}$ and $3 \mathrm{hr}$ at $25{ }^{\circ} \mathrm{C}$, with mixing using rotator shaker (120rpm), all the tubes were centrifuged for $10 \mathrm{~min}$ at $1500 \mathrm{rpm}$ and the supernatant was decanted carefully into a clean tube and then AFs measured by HPLC.

\section{In vivo study}

\section{Preparation of aflatoxins dose}

The DMSO containing AFs solution consisted of a mixture of $\mathrm{AFB}_{1}, \mathrm{AFB}_{2}, \mathrm{AFG}_{1}$ and $\mathrm{AFG}_{2}$ at a total concentration of $340 \mathrm{mg} / \mathrm{L}$ in the ratio of $8: 3: 2: 1$, respectively used to prepare 2 final concentrations of AFs and administrated by gavage to rats for 6 week, low AFs dose $(1 \mathrm{mg} / 6 \mathrm{ml} \mathrm{DMSO} / \mathrm{kg} /$ once weekly) and high AFs dose $(2 \mathrm{mg} / 6 \mathrm{ml} \mathrm{DMSO} / \mathrm{kg} /$ once weekly).

\section{Preparation of humic acid dose}

Enough amounts of the humic acid were suspended in water and administered orally by gavages to rats for 6 weeks at 2 dose concentration, low dose $(200 \mathrm{mg} / \mathrm{kg} / \mathrm{day})$ and high dose $(400 \mathrm{mg} / \mathrm{kg} /$ day $)$.

\section{Animal and treatment}

Forty-five male albino rats weighing about $140-150 \mathrm{~g}$ (provided by the Laboratory Animal Center, Faculty of Veterinary Medicine, Cairo University) were housed in stainless steel cages in animal house in Regional Center for Food and Feed, Agricultural Research Center, Ministry of Agriculture, Giza, Egypt under controlled light and temperature conditions (12-hr light/dark cycle, $22 \pm 2^{\mathrm{O}} \mathrm{C}$ ). During the acclimation period (1 week) and experimental period ( 6 weeks), the normal basal diet was supplied ad libitum. The basal diet consisted of casein $20 \%$, corn oil $10 \%$, cellulose (wooden fibers) $5 \%$, salt mixture $4 \%$, vitamin mixture $1 \%$ and starch $60 \%$ (Lane - Peter and Pearson, 1971).

Rats were divided into nine groups and treated for 6 week as follow: G1 (control): served as vehicle treated control (received $6 \mathrm{ml} \mathrm{DMSO} / \mathrm{kg}$ b.w. once/ week (by gavage)); G2 (low HA): received orally 200mg HA/ kg b.w daily $+6 \mathrm{ml} \mathrm{DMSO} / \mathrm{kg}$ b.w. once/ week; G3 (high HA): received orally 400mg HA/ kg b.w daily+6ml DMSO/ kg b.w. once/ week; G4 (low AFs): received orally low dose of AFs (1mg AFs suspended in $6 \mathrm{ml} \mathrm{DMSO} / \mathrm{kg}$ b.w) once/week; G5 (high AFs): received orally high dose of AFs (2mg AFs suspended in 6ml DMSO/ kg b.w) once/week; G6 (low HA +low AFs): received orally low dose of AFs once/week + low dose of HA daily; G7 (low HA+ high AFs): received orally high dose of AFs once/week + low dose of HA daily; G8 (high HA+ low AFs): received orally low dose 
of AFs once/week + high dose of HA daily and G9 (high HA+ high AFs): received orally high dose of AFs once/week + high dose of HA daily.

At the $3^{\text {rd }}$ week and $6^{\text {th }}$ week of the treatment period, blood samples were collected from the retro-orbital venous plexus under carbon dioxide anesthesia into two tubes. One contains heparin to obtain plasma. The second tube contains no anticoagulant to obtain serum. Plasma and serum separated and stored at $-20^{\circ} \mathrm{C}$ until analysis.

\section{Biochemical assays}

The ACE Alera Clinical Chemistry System Automatic Analyzer (Alfa Wasserman Corporation) was employed to measure the following parameters: ALT, AST, ALP, GGT, $\mathrm{TP}$, albumin, creatinine, urea, total cholesterol, triglycerides and glucose in serum.

\section{Estimation of antioxidant profile}

The extent of lipid peroxidation in terms of thiobarbituric acid reactive substances (TBARS) which react with malondialdehyde (MDA) in acidic medium was measured in serum calorimetrically at $534 \mathrm{~nm}$ according to the method of Onkawa et al. (1979). Plasma GSH was estimated calorimetrically at $405 \mathrm{~nm}$ based on the reduction of 5, 5 dithiobis (2nitrobenzoic acid) (DTNB) with GSH to produce yellow compound which directly proportional to GSH concentration (Beutler et al., 1963). Plasma GST was determined spectrophotometrically by measuring the conjugation of 1-chloro-2,4- dinitobenzene (CDNB) with reduced glutathione. The conjucation is accompined by an increase absorbance at $340 \mathrm{~nm}$. The rate of increase is directly proportional to the GST activity (Habig $\boldsymbol{e t}$ al., 1974). Plasma SOD activity was estimated spectrophotometrically at $560 \mathrm{~nm}$ based on the method described by Nishikimi et al. (1972) which relies on the ability of the enzyme to inhibite the phenazine methosulphate-mediated reduction of nitroblue tetrazolium dye.

\section{Statistical analysis:}

Statistical analysis of the obtained data was done using the least significant difference test (LSD) at the 5\% level of probability as outlined by Snedecor and Cochran (1980). Using the Duncan test institute program used a computer in the statistical analysis.

\section{RESULTS}

\section{In vitro results}

The in vitro adsorption capacity of HA for AFs in aqueous solution results illustrated in table (1) revealed that HA adsorb AFs with high affinity and adsorption not significantly affected by increasing interaction time, indicating that this was a fast adsorption process and the adsorption approach equilibrium in nearly $1 \mathrm{hr}$. Moreover, there was a dose-dependent response between HA concentration and amount of adsorbed AFs. While the affinity of HA to bind AFs was decreased by increasing AFs concentration. The addition of $100 \mathrm{mg}$ of HA to 20ppb AFs showed the highest bound \% (90.15-90.50\%) whereas, the addition of $20 \mathrm{mg}$ of HA to 100ppb AFs showed the lowest bound \% (80.83-80.87\%). 
Table (1): Amount of AFs adsorbed (ppb) and its percentage (\%) after different interaction times $(1 \mathrm{hr}, 2 \mathrm{hr}$ and $3 \mathrm{hr})$

\begin{tabular}{|c|c|c|c|c|}
\hline \multicolumn{2}{|c|}{ (mafs ppb } & 20ppb & 60ppb & $100 p p b$ \\
\hline \multirow{3}{*}{20} & $1 \mathrm{hr}$ & $\begin{array}{c}16.63 \\
83.15 \%\end{array}$ & $\begin{array}{c}49.60 \\
82.67 \%\end{array}$ & $\begin{array}{c}80.87 \\
80.87 \%\end{array}$ \\
\hline & $2 \mathrm{hr}$ & $\begin{array}{c}16.67 \\
83.35 \% \\
\end{array}$ & $\begin{array}{c}49.63 \\
82.72 \% \\
\end{array}$ & $\begin{array}{c}80.83 \\
80.83 \% \\
\end{array}$ \\
\hline & 3hr & $\begin{array}{c}16.77 \\
83.85 \%\end{array}$ & $\begin{array}{c}49.67 \\
82.78 \%\end{array}$ & $\begin{array}{c}80.83 \\
80.83 \%\end{array}$ \\
\hline \multirow{3}{*}{60} & $1 \mathrm{hr}$ & $\begin{array}{c}17.10 \\
85.50 \%\end{array}$ & $\begin{array}{c}50.17 \\
83.62 \%\end{array}$ & $\begin{array}{c}81.47 \\
81.47 \%\end{array}$ \\
\hline & $2 \mathrm{hr}$ & $\begin{array}{c}17.13 \\
85.65 \%\end{array}$ & $\begin{array}{c}50.33 \\
83.88 \%\end{array}$ & $\begin{array}{c}81.50 \\
81.50 \%\end{array}$ \\
\hline & $3 \mathrm{hr}$ & $\begin{array}{c}17.17 \\
85.85 \%\end{array}$ & $\begin{array}{c}50.37 \\
83.95 \%\end{array}$ & $\begin{array}{c}81.43 \\
81.43 \%\end{array}$ \\
\hline \multirow{3}{*}{100} & $1 \mathrm{hr}$ & $\begin{array}{c}18.07 \\
90.35 \%\end{array}$ & $\begin{array}{c}50.97 \\
84.95 \%\end{array}$ & $\begin{array}{c}82.23 \\
82.23 \%\end{array}$ \\
\hline & $2 \mathrm{hr}$ & $\begin{array}{c}18.10 \\
90.50 \%\end{array}$ & $\begin{array}{c}51.00 \\
85.00 \%\end{array}$ & $\begin{array}{c}82.30 \\
82.30 \%\end{array}$ \\
\hline & $3 \mathrm{hr}$ & $\begin{array}{c}18.03 \\
90.15 \%\end{array}$ & $\begin{array}{c}51.03 \\
85.05 \%\end{array}$ & $\begin{array}{c}82.33 \\
82.33 \%\end{array}$ \\
\hline
\end{tabular}

\section{In vivo results}

In this study, the data as shown in tables (2-6) recorded that, the administration of HA alone at both doses (G2 and G3) did not induce any obvious changes in all examined parameters except plasma GSH which elevated significantly along experimental periods (comparing to control (G1) $(P<0.05)$ ).

\section{Liver function parameters}

Table (2) show the effect of HA administration on liver functions in serum of rats treated with AFs. Analysis of variance indicated that there was a significant dose dependantincrease in ALT, AST, ALP and GGT activities, while, a significant dose dependantdecrease in TP and albumin concentrations was detected in rats treated with both AFs doses (G4 and G5) for 3 and 6 weeks. The HA administration significantly ameliorated the AFsinduced changes in liver functions as evidenced by a significant decrease of ALT, AST, ALP and GGT activities, and increase in TP and albumin concentration. The ameliorated effect of HA was more pronounced at high dose level and in low AFs treated groups (comparing with corresponding groups). Whereas, a substantial recovery in ALP, GGT activities, TP and albumin concentrations was observed in low AFs treated rats (G8) at $6^{\text {th }}$ week of treatment (compared with control (G1) $(P<0.05)$ ).

\section{Kidney function parameters:}

Results tabulated in table (3) demonstrated that oral AFs administration for period of 3 and 6 weeks at both 2 dose levels (rats of G4 and G5) resulted in a 
Table (2): Liver functions parameters in serum of control and treated rats during experimental periods (means $\pm \mathrm{SE}$ ).

\begin{tabular}{|c|c|c|c|c|c|c|c|c|c|c|c|c|}
\hline \multirow{2}{*}{ Groups } & \multicolumn{2}{|c|}{ ALT (U/L) } & \multicolumn{2}{|c|}{ AST (U/L) } & \multicolumn{2}{|c|}{$\operatorname{ALP}(\mathbf{U} / \mathbf{L})$} & \multicolumn{2}{|c|}{ GGT (U/L) } & \multicolumn{2}{|c|}{ TP ( g/dL) } & \multicolumn{2}{|c|}{ Albumin (g/dL) } \\
\hline & 3 weeks & weeks & 3 weeks & 6 weeks & 3 weeks & 6 weeks & 3 weeks & 6 weeks & 3 weeks & 6 weeks & 3 weeks & 6 weeks \\
\hline $\begin{array}{l}\text { G1 } \\
\text { Control }\end{array}$ & $\begin{array}{l}40.33 \\
\pm 0.89^{\mathrm{f}} \\
\end{array}$ & \pm 1.10 & $\pm 0.58^{\mathrm{e}}$ & \pm 0.88 & & $\begin{array}{c}408.00 \\
\pm 10.69^{\mathrm{fg}}\end{array}$ & $\begin{array}{c}2.67 \\
\pm 0.33^{\mathrm{e}} \\
\end{array}$ & & & & & \\
\hline $\begin{array}{l}\text { G2 } \\
\text { Low HA }\end{array}$ & $\begin{array}{l}40.67 \\
\pm 1.45^{\mathrm{f}} \\
\end{array}$ & $\begin{array}{l}1.33 \\
0.88^{\mathrm{f}} \\
\end{array}$ & $\begin{array}{l}86.33^{\mathrm{e}} \\
\pm 0.88 \\
\end{array}$ & $5^{\mathrm{f}}$ & \pm 7 & $\pm 9.07^{\mathrm{g}}$ & $\begin{array}{c}2.67 \\
\pm 0.33^{\mathrm{e}}\end{array}$ & $\begin{array}{c}3.33 \\
\pm .033^{\mathrm{e}}\end{array}$ & $\begin{array}{c}8.10 \\
\pm 0.13^{\mathrm{a}}\end{array}$ & $\begin{array}{c}8.07 \\
\pm 0.07^{\mathrm{a}}\end{array}$ & $\begin{array}{c}4.39 \\
\pm 0.04^{\mathrm{a}}\end{array}$ & $\begin{array}{c}4.41 \\
\pm 0.06^{\mathrm{a}}\end{array}$ \\
\hline $\begin{array}{l}\text { G3 } \\
\text { High HA }\end{array}$ & & & & & & & $\begin{array}{r}2 . \\
\pm 0 .\end{array}$ & $\begin{array}{r}3 \\
\pm 0 \\
\end{array}$ & $\begin{array}{r}8 . \\
\pm 0 \\
\end{array}$ & $\begin{array}{r}8 \\
\pm 0 \\
\end{array}$ & $\begin{array}{r}4 \\
\pm 0 \\
\end{array}$ & $\begin{array}{r}4 \\
\pm 0 \\
\end{array}$ \\
\hline $\begin{array}{l}\text { G4 } \\
\text { Low AFs }\end{array}$ & & & & & & $\begin{array}{r}69 \\
\pm 1( \\
\end{array}$ & $\begin{array}{r}8 . \\
\pm 0 .\end{array}$ & $\begin{array}{r}9 . \\
\pm 0 \\
\end{array}$ & $\begin{array}{r}5 . \\
\pm 0\end{array}$ & $\begin{array}{r}5 . \\
\pm 0\end{array}$ & $\begin{array}{c}3.53 \\
\pm 0.05^{\mathrm{d}} \\
\end{array}$ & $\begin{array}{c}3.12 \\
\pm 0.05^{\mathrm{c}} \\
\end{array}$ \\
\hline $\begin{array}{l}\text { G5 } \\
\text { High AFs }\end{array}$ & & & $\pm 1.20^{\mathrm{a}}$ & $\pm 1.73^{\mathrm{a}}$ & & 0 & $\begin{array}{r}10.66 \\
\pm 0.33^{\mathrm{a}} \\
\end{array}$ & $\begin{array}{r}11.33 \\
\pm 0.33^{\mathrm{a}} \\
\end{array}$ & $\begin{array}{c}5.01 \\
\pm 0.05^{\mathrm{f}}\end{array}$ & $\begin{array}{r}4.0 \\
\pm 0 .\end{array}$ & $\begin{array}{c}2.84 \\
\pm 0.03^{\mathrm{f}} \\
\end{array}$ & $\begin{array}{c}2.26 \\
\pm 0.04^{\mathrm{d}}\end{array}$ \\
\hline $\begin{array}{l}\text { G6 } \\
\text { Low HA } \\
+ \text { Low AFs } \\
\end{array}$ & $\begin{array}{c}59.00 \\
\pm 0.58^{d}\end{array}$ & $\begin{array}{l}53.00 \\
\pm 0.58^{\mathrm{d}}\end{array}$ & $\begin{array}{l}122.67 \\
\pm 2.03^{c}\end{array}$ & $\begin{array}{l}112.67 \\
\pm 1.76^{\mathrm{d}}\end{array}$ & $\begin{array}{l}508.33 \\
\pm 3.38^{\mathrm{c}}\end{array}$ & $\begin{array}{l}57.33 \\
5.78^{\mathrm{e}}\end{array}$ & $\begin{array}{c}6.67 \\
\pm 0.33^{\mathrm{c}}\end{array}$ & $\begin{array}{c}5.67 \\
\pm 0.33^{\mathrm{d}}\end{array}$ & $\begin{array}{c}6.94 \\
\pm 0.04^{\mathrm{c}}\end{array}$ & $\begin{array}{c}7.22 \\
\pm 0.06^{\mathrm{b}}\end{array}$ & $\begin{array}{c}3.83 \\
\pm 0.04^{\mathrm{c}}\end{array}$ & $\begin{array}{c}3.95 \\
\pm 0.05^{\mathrm{b}}\end{array}$ \\
\hline $\begin{array}{l}\text { G7 } \\
\text { Low HA + } \\
\text { High AFs }\end{array}$ & $\begin{array}{c}66.33 \\
\pm 0.88^{\mathrm{b}}\end{array}$ & $\begin{array}{r}79.00 \\
\pm 1.52^{\mathrm{b}}\end{array}$ & $\begin{array}{l}131.33 \\
\pm 1.20^{\mathrm{b}}\end{array}$ & $\begin{array}{l}123.33 \\
\pm 1.20^{\mathrm{c}}\end{array}$ & $\begin{array}{l}592.67 \\
\pm 5.04^{\mathrm{b}}\end{array}$ & $\begin{array}{l}568.33 \\
\pm 2.33^{\mathrm{c}}\end{array}$ & $\begin{array}{c}8.33 \\
\pm 0.33^{\mathrm{b}}\end{array}$ & $\begin{array}{c}7.33 \\
\pm 0.33^{\mathrm{c}}\end{array}$ & $\begin{array}{c}6.06 \\
\pm 0.04^{\mathrm{e}}\end{array}$ & $\begin{array}{c}6.29 \\
\pm 0.04^{\mathrm{d}}\end{array}$ & $\begin{array}{c}2.99 \\
\pm 0.03^{\mathrm{e}}\end{array}$ & $\begin{array}{c}3.16 \\
\pm 0.04^{\mathrm{c}}\end{array}$ \\
\hline $\begin{array}{l}\text { G8 } \\
\text { High HA + } \\
\text { Low AFs }\end{array}$ & $\begin{array}{l}55.67 \\
\pm 0.33^{\mathrm{e}}\end{array}$ & $\begin{array}{l}48.00 \\
\pm 0.58^{\mathrm{e}}\end{array}$ & $\begin{array}{l}115.00 \\
\pm 1.53^{\mathrm{d}}\end{array}$ & $\begin{array}{l}100.00 \\
\pm 0.58^{\mathrm{e}}\end{array}$ & $\begin{array}{l}467.67 \\
\pm 5.21^{\mathrm{d}}\end{array}$ & $\begin{array}{l}430.33 \\
\pm 4.41^{\mathrm{f}}\end{array}$ & $\begin{array}{c}4.67 \\
\pm 0.33^{\mathrm{d}}\end{array}$ & $\begin{array}{c}4.33 \\
\pm 0.33^{\mathrm{e}}\end{array}$ & $\begin{array}{c}7.57 \\
\pm 0.06^{\mathrm{b}}\end{array}$ & $\begin{array}{c}7.99 \\
\pm 0.03^{\mathrm{a}}\end{array}$ & $\begin{array}{c}4.18 \\
\pm 0.04^{\mathrm{b}}\end{array}$ & $\begin{array}{c}4.35 \\
\pm 0.05^{\mathrm{a}}\end{array}$ \\
\hline $\begin{array}{l}\text { G9 } \\
\text { High HA } \\
\text { +High AF }\end{array}$ & $\begin{array}{c}61.67 \\
\pm 1.45^{\mathrm{cd}}\end{array}$ & $\begin{array}{c}56 \\
\pm 0.58^{\mathrm{d}}\end{array}$ & $\begin{array}{l}122.66 \\
\pm 0.88^{c}\end{array}$ & $\begin{array}{l}111.67 \\
\pm 2.02^{\mathrm{d}}\end{array}$ & $\begin{array}{l}522 \\
\pm 4\end{array}$ & $\begin{array}{l}67 \\
9^{d}\end{array}$ & $\begin{array}{c}6.33 \\
\pm 0.33^{\mathrm{c}}\end{array}$ & $\begin{array}{c}5.33 \\
\pm 0.33^{\mathrm{d}}\end{array}$ & $\begin{array}{c}6.65 \\
\pm 0.18^{\mathrm{d}}\end{array}$ & $\begin{array}{c}6.93 \\
\pm 0.06^{\mathrm{c}}\end{array}$ & $\begin{array}{c}3.79 \\
\pm 0.02^{\mathrm{c}}\end{array}$ & $\begin{array}{c}4.02 \\
\pm 0.02^{\mathrm{b}}\end{array}$ \\
\hline $\mathbf{L S D}_{0.05}$ & 2.801 & 3.079 & 35 & 291 & 5.125 & 21.353 & .990 & .990 & 0.257 & .202 & 0.117 & 0.136 \\
\hline
\end{tabular}

Within the same column, various superscript letters indicate significant differences (Duncan, $P<0.05$ ). 
Table (3): Kidney functions parameters in serum of control and treated rats during experimental periods (means $\pm \mathrm{SE}$ ).

\begin{tabular}{|c|c|c|c|c|}
\hline \multirow{2}{*}{ Groups } & \multicolumn{2}{|c|}{ Creatinine (mg/dL) } & \multicolumn{2}{|c|}{ Urea (mg/dL) } \\
\hline & 3 weeks & 6 weeks & 3 weeks & 6 weeks \\
\hline $\begin{array}{l}\text { G1 } \\
\text { Control }\end{array}$ & $0.48 \pm 0.01^{\mathrm{e}}$ & $0.50 \pm 0.01^{\mathrm{e}}$ & $35.00 \pm 1.16^{\mathrm{f}}$ & $37.00 \pm 1.16^{\mathrm{ef}}$ \\
\hline $\begin{array}{l}\text { G2 } \\
\text { Low HA }\end{array}$ & $0.49 \pm 0.01^{\mathrm{e}}$ & $0.50 \pm 0.01^{\mathrm{e}}$ & $35.67 \pm 0.88^{f}$ & $37.00 \pm 1.00^{\mathrm{ef}}$ \\
\hline $\begin{array}{l}\text { G3 } \\
\text { High HA }\end{array}$ & $0.49 \pm 0.02^{\mathrm{e}}$ & $0.49 \pm 0.01^{\mathrm{e}}$ & $34.67 \pm 0.88^{\mathrm{f}}$ & $36.67 \pm 0.88^{\mathrm{f}}$ \\
\hline $\begin{array}{l}\text { G4 } \\
\text { Low AFs }\end{array}$ & $0.60 \pm 0.02^{\mathrm{c}}$ & $0.69 \pm 0.01^{\mathrm{b}}$ & $53.33 \pm 0.88^{c}$ & $63.33 \pm 0.88^{b}$ \\
\hline $\begin{array}{l}\text { G5 } \\
\text { High AFs }\end{array}$ & $0.72 \pm 0.2^{\mathrm{a}}$ & $0.82 \pm 0.02^{\mathrm{a}}$ & $63.67 \pm 1.20^{\mathrm{a}}$ & $72.00 \pm 1.73^{\mathrm{a}}$ \\
\hline $\begin{array}{l}\text { G6 } \\
\text { Low HA } \\
+ \text { Low AFs }\end{array}$ & $0.55 \pm 0.01^{\mathrm{d}}$ & $0.52 \pm 0.02^{\mathrm{de}}$ & $49.67 \pm 0.88^{d}$ & $44.00 \pm 0.58^{\mathrm{d}}$ \\
\hline $\begin{array}{l}\text { G7 } \\
\text { Low HA } \\
+ \text { High AFs }\end{array}$ & $0.68 \pm 0.01^{\mathrm{b}}$ & $0.59 \pm 0.01^{\mathrm{c}}$ & $60.00 \pm 0.58^{b}$ & $54.67 \pm 0.88^{\mathrm{c}}$ \\
\hline $\begin{array}{l}\text { G8 } \\
\text { High HA } \\
+ \text { Low AFs }\end{array}$ & $0.52 \pm 0.01^{\mathrm{e}}$ & $0.50 \pm 0.04^{\mathrm{e}}$ & $43.33 \pm 0.88^{\mathrm{e}}$ & $40.00 \pm 0.58^{\mathrm{e}}$ \\
\hline $\begin{array}{l}\text { G9 } \\
\text { High HA } \\
\text { +High AFs }\end{array}$ & $0.58 \pm 0.01^{\mathrm{cd}}$ & $0.54 \pm 0.003^{\mathrm{d}}$ & $50.33 \pm 0.88^{d}$ & $45.67 \pm 0.88^{d}$ \\
\hline LSD $_{0.05}$ & 0.036 & 0.036 & 2.762 & 2.989 \\
\hline
\end{tabular}

Within the same column, various superscript letters indicate significant differences (Duncan, $P<0.05$ ).

significant dose-dependent elevation $(P<0.05)$ of serum creatinine and urea concentration. However, supplementation of AFs-intoxicated rats with HA (G6, G7, G8 and G9) ameliorated the adverse effect of both AFs doses especially at low AFs dose. Whereas, high HA dose administration restore creatinine and urea concentration to normal in serum of rats treated with low AFs dose (G8) along treatment period and at $6^{\text {th }}$ week of treatment, respectively (comparing with control $(P<0.05)$ ).

\section{Lipid profile parameters:}

The data in table (4) shows that AFs induced significant dose-dependent increase in serum total cholesterol and triglycerides concentrations comparing with control $(P<0.05)$ at all experimental periods. Simultaneous administrations of HA and AFs in G6, G7, G8 and G9 rats resulted in significant reduction of increased total cholesterol and triglycerides concentrations comparing with corresponding AFs-treated rats (G4 and G5). The best response was obtained by high HA dose. The high HA dose treatment was able to restore triglycerides level to normal at $6^{\text {th }}$ week of treatment in serum of low AFs-treated rats comparing with control $(P<0.05)$. 
Table (4): Total cholesterol and triglycerides concentrations in serum of control and treated rats during experimental periods (means $\pm \mathrm{SE}$ ).

\begin{tabular}{|c|c|c|c|c|}
\hline \multirow{2}{*}{ Groups } & \multicolumn{2}{|c|}{ Cholesterol (mg/dL) } & \multicolumn{2}{|c|}{ Triglycerides (mg/dL) } \\
\hline & 3 weeks & 6 weeks & 3 weeks & 6 weeks \\
\hline $\begin{array}{l}\text { G1 } \\
\text { Control }\end{array}$ & $37.00 \pm 1.00^{\mathrm{e}}$ & $39.00 \pm 1.16^{\mathrm{g}}$ & $82.33 \pm 1.45^{\mathrm{f}}$ & $89.33 \pm 3.53^{\mathrm{e}}$ \\
\hline $\begin{array}{l}\text { G2 } \\
\text { Low HA }\end{array}$ & $35.33 \pm 1.20^{\mathrm{e}}$ & $39.00 \pm 1.53^{\mathrm{g}}$ & $81.33 \pm 0.88^{\mathrm{f}}$ & $88.33 \pm 0.88^{\mathrm{e}}$ \\
\hline $\begin{array}{l}\text { G3 } \\
\text { High HA }\end{array}$ & $35.33 \pm 1.45^{\mathrm{e}}$ & $38.33 \pm 1.20^{\mathrm{g}}$ & $81.33 \pm 1.45^{\mathrm{f}}$ & $88.33 \pm 1.76^{\mathrm{e}}$ \\
\hline $\begin{array}{l}\text { G4 } \\
\text { Low AFs }\end{array}$ & $71.67 \pm 1.45^{b}$ & $91.33 \pm 1.45^{\mathrm{b}}$ & $127.33 \pm 2.03^{c}$ & $149.33 \pm 3.53^{b}$ \\
\hline $\begin{array}{l}\text { G5 } \\
\text { High AFs }\end{array}$ & $90.67 \pm 2.73^{a}$ & $121.00 \pm 5.57^{\mathrm{a}}$ & $164.33 \pm 1.20^{\mathrm{a}}$ & $206.33 \pm 6.89^{a}$ \\
\hline $\begin{array}{l}\text { G6 } \\
\text { Low HA } \\
+ \text { Low AFs }\end{array}$ & $59.33 \pm 1.20^{\mathrm{c}}$ & $50.33 \pm 0.88^{\mathrm{e}}$ & $118.67 \pm 0.88^{\mathrm{d}}$ & $105.33 \pm 3.76^{\mathrm{d}}$ \\
\hline $\begin{array}{l}\text { G7 } \\
\text { Low HA } \\
+ \text { High AFs }\end{array}$ & $70.00 \pm 1.16^{\mathrm{b}}$ & $62.00 \pm 1.16^{\mathrm{c}}$ & $147.67 \pm 1.86^{\mathrm{b}}$ & $140.00 \pm 1.56^{\mathrm{b}}$ \\
\hline $\begin{array}{l}\text { G8 } \\
\text { High HA } \\
\text { + Low AFs }\end{array}$ & $51.67 \pm 0.88^{d}$ & $43.67 \pm 0.67^{f}$ & $110.00 \pm 1.53^{\mathrm{e}}$ & $98.33 \pm 0.88^{\mathrm{de}}$ \\
\hline $\begin{array}{l}\text { G9 } \\
\text { High HA } \\
\text { +High AFs }\end{array}$ & $62.33 \pm 2.19^{c}$ & $55.67 \pm 0.88^{d}$ & $128.00 \pm 2.08^{\mathrm{c}}$ & $119.33 \pm 0.88^{c}$ \\
\hline $\mathbf{L S D}_{0.05}$ & 4.692 & 4.253 & 4.586 & 9.562 \\
\hline
\end{tabular}

Within the same column, various superscript letters indicate significant differences (Duncan, $P<0.05$ ).

Glucose concentration:

Oral administration of AFs for $6^{\text {th }}$ weeks caused a significant dose-dependent increase in serum glucose level in respect to control group $(P<0.05)$. The HA supplementation significantly ameliorated hyperglycemic effect of AFs in comparison with AFs treated groups (table 5) but it failed to normalize glucose level. Such effect was more pronounced with low AFs dose and seemed to be HA dose - dependent.

\section{Antioxidant profile:}

Table (6) demonstrates that AFs treatment significantly reduce $(P<0.05)$ plasma GSH, GST and SOD levels in dose-dependent manner in all experimental periods. The depleted level of GSH, GST and SOD was elevated significantly by HA administration especially with low AFs dose comparing with corresponding AFs group $(P<0.05)$. This elevation was seemed to be parallel to time of treatment and dose of HA. Furthermore high HA intake (G8) restores GSH at $3^{\text {rd }}$ week and GST and SOD at $6^{\text {th }}$ week of treatment to normal in plasma of low AFs-intoxicated rats comparing with control group $(\mathrm{G} 1)(P<0.05)$. It worthy to note that high HA intake enhance GSH production and increase its level than normal at $6^{\text {th }}$ week of treatment in respect to control group $(P<0.05)$. 
Table (5): Glucose concentrations in serum of control and treated rats during experimental periods (means $\pm \mathrm{SE}$ ).

\begin{tabular}{|l|c|c|}
\hline \multirow{2}{*}{ Parameter } & \multicolumn{2}{|c|}{ Glucose (mg/dL) } \\
\cline { 2 - 3 } Groups & 3 weeks & 6 weeks \\
\hline $\begin{array}{l}\text { Gontrol } \\
\text { Low HA }\end{array}$ & $89.33 \pm 1.45^{\mathrm{f}}$ & $99.68 \pm 0.88^{\mathrm{g}}$ \\
\hline $\begin{array}{l}\text { G3 } \\
\text { High HA }\end{array}$ & $91.33 \pm 1.76^{\mathrm{f}}$ & $101.33 \pm 1.20^{\mathrm{g}}$ \\
\hline $\begin{array}{l}\text { G4 } \\
\text { Low AFs }\end{array}$ & $87.67 \pm 1.20^{\mathrm{f}}$ & $98.33 \pm 0.88^{\mathrm{g}}$ \\
\hline $\begin{array}{l}\text { G5 } \\
\text { High AFs }\end{array}$ & $147.33 \pm 0.88^{\mathrm{b}}$ & $160.67 \pm 0.88^{\mathrm{b}}$ \\
\hline $\begin{array}{l}\text { G6 } \\
\text { Low HA + Low AFs }\end{array}$ & $130.00 \pm 1.56^{\mathrm{a}}$ & $178.00 \pm 1.53^{\mathrm{a}}$ \\
\hline $\begin{array}{l}\text { G7 } \\
\text { Low HA + High AFs }\end{array}$ & $149.33 \pm 145^{\mathrm{b}}$ & $120.33 \pm 0.88^{\mathrm{e}}$ \\
\hline $\begin{array}{l}\text { G8 } \\
\text { High HA + Low AFs }\end{array}$ & $125.00 \pm 1.00^{\mathrm{e}}$ & $140.33 \pm 1.45^{\mathrm{c}}$ \\
\hline $\begin{array}{l}\text { G9 } \\
\text { High HA +High AFs }\end{array}$ & $138.00 \pm 1.16^{\mathrm{c}}$ & $110.33 \pm 2.73^{\mathrm{f}}$ \\
\hline LSD & 3.05 & $127.00 \pm 1.00^{\mathrm{d}}$ \\
\hline Win & 3.136 \\
\hline
\end{tabular}

Within the same column, various superscript letters indicate significant differences (Duncan, $P<0.05$ ).

Lipid peroxidation was estimated from measured MDA formation (table 7) in order to evaluate the effect of HA on AFs-induced oxidative injury. The AFs significantly increased $(P<0.05)$ serum MDA levels in dose- dependent manner in comparison to control. However, HA treatment at both doses significantly minimizes MDA production (comparing with AFs groups). Such effect was more pronounced with low AFs dose and seemed to be time and HA dose-dependent.

\section{DISCUSSION}

Contamination with AFs is responsible for significant financial losses that encompass a broad spectrum of crop and farm animals and extends through the food chain to humans. The results of this study indicate that HA has a high affinity to adsorb AFs. The adsorption affinity was increased by elevation HA concentration and decreasing AFs concentration. Adsorption not significantly affected by increasing interaction time, indicating that this was a fast adsorption process and the adsorption approach equilibrium in nearly $1 \mathrm{hr}$. The same pattern of adsorption was previously reported by Ye et al. (2009) which demonstrate that sodium humate has the potential as an $\mathrm{AFB}_{1}$ adsorbent. Sodium humate has several advantages on $\mathrm{AFB}_{1}$ adsorption: i) higher affinity to $\mathrm{AFB}_{1}$; ii) not adsorb other nutrients; iii) the complex was very stable in different $\mathrm{PH}$ phosphate buffer. According to Ramos and Herna'ndez (1996), this might indicate the presence of adsorption centers within the HA with different affinities for $\mathrm{AF}$, resulting in a heterogeneous adsorbent surface or the coexistence of different adsorption mechanisms. 
Table (6): Antioxidants parameters in plasma of control and treated rats during experimental periods (means $\pm \mathrm{SE}$ ).

\begin{tabular}{|c|c|c|c|c|c|c|}
\hline \multirow{2}{*}{ Groups } & \multicolumn{2}{|c|}{$\begin{array}{c}\text { GSH-reduced } \\
(\mathrm{mg} / \mathrm{dL})\end{array}$} & \multicolumn{2}{|c|}{ GST (u/ml) } & \multicolumn{2}{|c|}{$\operatorname{SOD}(\mathbf{u} / \mathbf{m l})$} \\
\hline & 3 weeks & 6 weeks & 3 weeks & 6 weeks & 3 weeks & 6 weeks \\
\hline $\begin{array}{l}\text { G1 } \\
\text { Control }\end{array}$ & $\begin{array}{c}0.62 \\
\pm 0.009^{b}\end{array}$ & $\begin{array}{c}0.65 \\
\pm 0.015^{\mathrm{b}}\end{array}$ & $\begin{array}{l}68.91 \\
\pm 0.88^{\mathrm{a}}\end{array}$ & $\begin{array}{c}69.98 \\
\pm 0.99^{\mathrm{a}}\end{array}$ & $\begin{array}{l}555.00 \\
\pm 7.59^{\mathrm{a}}\end{array}$ & $\begin{array}{l}560.97 \\
\pm 3.88^{a}\end{array}$ \\
\hline $\begin{array}{l}\text { G2 } \\
\text { Low HA }\end{array}$ & $\begin{array}{c}0.64 \\
\pm 0.003^{\mathrm{a}}\end{array}$ & $\begin{array}{c}0.69 \\
\pm 0.006^{\mathrm{a}}\end{array}$ & $\begin{array}{r}70.34 \\
\pm 0.72^{\mathrm{a}}\end{array}$ & $\begin{array}{c}70.83 \\
\pm 1.60^{\mathrm{a}}\end{array}$ & $\begin{array}{l}551.49 \\
\pm 8.05^{\mathrm{a}}\end{array}$ & $\begin{array}{l}559.31 \\
\pm 4.59^{\mathrm{a}}\end{array}$ \\
\hline $\begin{array}{l}\text { G3 } \\
\text { High HA }\end{array}$ & $\begin{array}{c}0.65 \\
\pm 0.004^{\mathrm{a}}\end{array}$ & $\begin{array}{c}0.71 \\
\pm 0.009^{\mathrm{a}}\end{array}$ & $\begin{array}{r}69.93 \\
\pm 1.27^{\mathrm{a}}\end{array}$ & $\begin{array}{c}70.81 \\
\pm 0.65^{\mathrm{a}}\end{array}$ & $\begin{array}{l}553.90 \\
\pm 8.28^{\mathrm{a}}\end{array}$ & $\begin{array}{l}561.93 \\
\pm 3.47^{\mathrm{a}}\end{array}$ \\
\hline $\begin{array}{l}\text { G4 } \\
\text { Low AFs }\end{array}$ & $\begin{array}{c}0.41 \\
\pm 0.012^{\mathrm{f}}\end{array}$ & $\begin{array}{c}0.30 \\
\pm 0.012^{\mathrm{e}}\end{array}$ & $\begin{array}{l}54.02 \\
\pm 0.99^{d}\end{array}$ & $\begin{array}{l}46.36 \\
\pm 1.44^{\mathrm{d}}\end{array}$ & $\begin{array}{l}415.94 \\
\pm 3.58^{\mathrm{d}}\end{array}$ & $\begin{array}{l}369.94 \\
\pm 8.94^{\mathrm{d}}\end{array}$ \\
\hline $\begin{array}{l}\text { G5 } \\
\text { High AFs }\end{array}$ & $\begin{array}{c}0.27 \\
\pm 0.009^{\mathrm{g}}\end{array}$ & $\begin{array}{c}0.16 \\
\pm 0.009^{\mathrm{f}}\end{array}$ & $\begin{array}{l}42.86 \\
\pm 0.64^{\mathrm{f}}\end{array}$ & $\begin{array}{c}36.29 \\
\pm 1.12^{\mathrm{e}}\end{array}$ & $\begin{array}{l}345.19 \\
\pm 4.42^{\mathrm{e}}\end{array}$ & $\begin{array}{l}245.88 \\
\pm 9.47^{\mathrm{e}}\end{array}$ \\
\hline $\begin{array}{l}\text { G6 } \\
\text { Low HA } \\
+ \text { Low AFs }\end{array}$ & $\begin{array}{c}0.52 \\
\pm 0.009^{\mathrm{d}}\end{array}$ & $\begin{array}{c}0.60 \\
\pm 0.013^{\mathrm{c}}\end{array}$ & $\begin{array}{l}59.60 \\
\pm 0.69^{c}\end{array}$ & $\begin{array}{c}63.80 \\
\pm 0.44^{\mathrm{b}}\end{array}$ & $\begin{array}{l}489.67 \\
\pm 5.08^{c}\end{array}$ & $\begin{array}{l}514.18 \\
\pm 3.78^{\mathrm{b}}\end{array}$ \\
\hline $\begin{array}{l}\text { G7 } \\
\text { Low HA } \\
+ \text { High AFs }\end{array}$ & $\begin{array}{c}0.46 \\
\pm 0.012^{\mathrm{e}}\end{array}$ & $\begin{array}{c}0.54 \\
\pm 0.006^{\mathrm{d}}\end{array}$ & $\begin{array}{c}50.49 \\
\pm 0.49^{\mathrm{e}}\end{array}$ & $\begin{array}{c}55.98 \\
\pm 0.78^{c}\end{array}$ & $\begin{array}{l}421.04 \\
\pm 5.04^{\mathrm{d}}\end{array}$ & $\begin{array}{l}452.53 \\
\pm 6.19^{c}\end{array}$ \\
\hline $\begin{array}{l}\text { G8 } \\
\text { High HA } \\
+ \text { Low AFs }\end{array}$ & $\begin{array}{c}0.61 \\
\pm 0.003^{\mathrm{b}}\end{array}$ & $\begin{array}{c}0.69 \\
\pm 0.007^{\mathrm{a}}\end{array}$ & $\begin{array}{c}64.93 \\
\pm 0.95^{\mathrm{b}}\end{array}$ & $\begin{array}{l}68.06 \\
\pm 0.89^{\mathrm{a}}\end{array}$ & $\begin{array}{l}522.52 \\
\pm 6.38^{\mathrm{b}}\end{array}$ & $\begin{array}{l}547.50 \\
\pm 2.96^{\mathrm{a}}\end{array}$ \\
\hline $\begin{array}{l}\text { G9 } \\
\text { High HA +High } \\
\text { AFs }\end{array}$ & $\begin{array}{c}0.54 \\
\pm 0.009^{c}\end{array}$ & $\begin{array}{c}0.63 \\
\pm 0.018^{\mathrm{bc}}\end{array}$ & $\begin{array}{l}58.27 \\
\pm 1.23^{\mathrm{c}}\end{array}$ & $\begin{array}{c}62.92 \\
\pm 0.95^{\mathrm{b}}\end{array}$ & $\begin{array}{l}482.15 \\
\pm 4.80^{\mathrm{c}}\end{array}$ & $\begin{array}{l}507.88 \\
\pm 6.06^{\mathrm{b}}\end{array}$ \\
\hline $\mathbf{L S D}_{0.05}$ & 0.026 & 0.033 & 2.725 & 3.261 & 18.215 & 17.603 \\
\hline
\end{tabular}

Within the same column, various superscript letters indicate significant differences (Duncan, $P<0.05$ ).

Where, HA have a tridimensional reticulate structure and contain multiple functional groups such as hydroxyl, carboxylic, carbonyl, amino, amido and sulphhydryl groups (MacCarthy, 2001). Due to different HA structures, the content of functional groups and various qualities (colloidal, spectral, electrochemical and ion exchange) their considerable adsorption capacity is assumed (Klocking, 1994 and Alvarez Puebla et al., 2005).

AFs are mutagenic, hepatotoxic and hepatocarcinogenic both for humans and animals and cause oxidative stress (Meki et al., 2004). Even small amounts of AF are dangerous for animal health because of detrimental effects on some biochemical parametrs (Keçeci $\boldsymbol{e t}$ al., 1998).

Data presented herein showed that treatment with AFs was found to alter all of the biochemical parameters. The serum levels of hepatic enzymes primarily reflect the degree of liver damage and have been commonly used as a diagnostic marker for hepatotoxicity (Pumford et al., 1997 and Ebadollahi-Natanzi et al., 2010). The administration of AFs for 6 weeks induced dose-dependent increase in ALT, AST, ALP and GGT activities and significant dose dependant-decrease in TP and albumin. 
Table (7): Lipid peroxidation (Malondialdehyde levels) in serum of control and treated rats during experimental periods (means $\pm \mathrm{SE}$ ).

\begin{tabular}{|c|c|c|}
\hline \multirow{2}{*}{$\begin{array}{l}\text { Parameter } \\
\text { Groups }\end{array}$} & \multicolumn{2}{|c|}{ MDA nmol/ml } \\
\hline & 3 weeks & 6 weeks \\
\hline $\begin{array}{l}\text { G1 } \\
\text { Control }\end{array}$ & $1.67 \pm 0.17^{\mathrm{f}}$ & $2.03 \pm 0.11^{\mathrm{f}}$ \\
\hline $\begin{array}{l}\text { G2 } \\
\text { Low HA }\end{array}$ & $1.60 \pm 0.15^{\mathrm{f}}$ & $1.90 \pm 0.23^{\mathrm{f}}$ \\
\hline $\begin{array}{l}\text { G3 } \\
\text { High HA }\end{array}$ & $1.86 \pm 0.19^{\mathrm{f}}$ & $1.92 \pm 0.11^{\mathrm{f}}$ \\
\hline $\begin{array}{l}\text { G4 } \\
\text { Low AFs }\end{array}$ & $8.52 \pm 0.07^{b}$ & $11.20 \pm 0.35^{\mathrm{b}}$ \\
\hline $\begin{array}{l}\text { G5 } \\
\text { High AFs }\end{array}$ & $9.89 \pm 0.19^{\mathrm{a}}$ & $17.19 \pm 1.17^{\mathrm{a}}$ \\
\hline $\begin{array}{l}\text { G6 } \\
\text { Low HA + Low AFs }\end{array}$ & $7.24 \pm 0.12^{\mathrm{d}}$ & $6.52 \pm 0.16^{\mathrm{d}}$ \\
\hline $\begin{array}{l}\text { G7 } \\
\text { Low HA + High AFs }\end{array}$ & $8.47 \pm 0.21^{\mathrm{b}}$ & $7.91 \pm 0.04^{\mathrm{c}}$ \\
\hline $\begin{array}{l}\text { G8 } \\
\text { High HA + Low AFs }\end{array}$ & $6.21 \pm 0.15^{\mathrm{e}}$ & $5.07 \pm 0.22^{\mathrm{e}}$ \\
\hline $\begin{array}{l}\text { G9 } \\
\text { High HA +High AFs }\end{array}$ & $7.77 \pm 0.17^{\mathrm{c}}$ & $6.87 \pm 0.08^{\mathrm{cd}}$ \\
\hline $\mathbf{L S D}_{0.05}$ & 0.480 & 1.272 \\
\hline
\end{tabular}

Within the same column, various superscript letters indicate significant differences (Duncan, $P<0.05$ ).

The increase in AST and ALT activity indicated initial hepatocellular damage (AbdelWahhab et al., 2006). Similar to our results, Banu et al. (2009); Preetha et al. (2006) reported an increase in ALT, AST and ALP in rats treated orally with AFB $_{1}(1 \mathrm{mg} / \mathrm{kg} \mathrm{b.w})$, suggesting hepatic damage; El-Nekeety et al. (2011); Nayak and Sashidhar (2010); Saleh et al. (2007) and Abdel-Wahhab et al. (2002) reported increase in liver function enzymes (ALT, AST, ALP and GGT) and decrease in TP and albumin in rat fed $\mathrm{AFB}_{1}$-contaminated diet. Such noticed increase in liver enzymes can be attributed to cell necrosis, changes in cell membrane permeability or impairment of biliary excretion (Pozzi et al., 2000). The decrease in plasma protein may be due to degeneration of endoplasmic reticulum and inhibition of protein synthesis (Terao and Ueno, 1978 and Srivastava, 1984). In addition, Osuna and Edds (1982) suggested that such effect may be due to the metabolism of AFs in liver, where it interferes with protein synthesis and RNA production, resulting in decreasing of albumin. Furthermore, in circulation, AF binds with plasma proteins especially albumin to form AF albumin adduct (Autrup et al. 1991). Moreover, the low level of TP may indicate protein catabolism and/or kidney dysfunctions (Abdel- Wahhab and Aly, 2003). ROS and consequent peroxidative damage caused by AFs are considered to be the main mechanisms leading to hepatotoxicity (Preetha et al., 2006) which proved by increased MDA concentration and decreased antioxidant enzymes.

Kidneys represent the major control system maintaining body homeostasis. The serum concentration of urea and creatinine determine renal function and are thus biomarkers for kidney disease (Levey et al., 1999). The present results revealed dose-dependent increase 
of creatinine and urea concentration in serum of AFs-intoxicated rats. Such finding was previously reported by Kanchana et al. (2013); El-Nekeety et al. (2011) and AbdelWahhab et al. (2002) in rats; El-Zahar et al. (1996) in rabbits. Taken together, these results clearly indicated that AFs have stressful effects on the hepatic and renal tissues, consistent with those reported in the literature of aflatoxicosis (Abdel-Wahhab and Kholif, 2008 and Sherif et al., 2009).

On the other hand, the significant dose-dependent increase in cholesterol and triglycerides in the group received the AFs alone are coincided with those reported previously in AFs-ingested animals (El-Nekeety et al., 2011; Edrington et al., 1995 and Abdel-Wahhab et al., 2006, 2007). This is probably associated with biliary obstruction and acute hepatic injury (Edrington et al., 1995).

In this experimental model, the oral poisoning of rats with AFs caused dosedependent hyperglycemia in all experimental period. Our result was in accordance with finding of Choi et al. (1981) and Rastogi et al. (2001) in rats; Abdelhamid et al. (1995) in chickens and Ankrah (1995) in mice. In rabbits, the addition of $\mathrm{AFB}_{1}$ to normal diet produced hyperglycemia and decreased liver glycogen (Verma and Raval, 1992). In this respect, the elevation of blood glucose level and the decrease in glycogen in liver by AF was attributed to lowering insulin level and rising of cortisol (Abdelhamid and Dorra, 1990).

The oxidative damage in a cell or tissue occurs when the concentration of ROS generated exceeds the antioxidant capability of the cell (Sies, 1991). Therefore, it could be due to significant decreases in the levels of non-enzymatic antioxidant (e.g. vitamin C, vitamin E, GSH) and enzymatic antioxidants (SOD, GST, glutathione peroxidase (GPx) and catalase (CAT)), which are the main determinants of the antioxidant defense mechanism of the cell. AF can produce reactive ROS including the superoxide anion, hydrogen peroxide, and the hydroxyl radical via cytochrome $P 450$ metabolism and an iron-mediated redox mechanism (Choi et al., 2010 and Preetha et al., 2006). ROS cause oxidative stress by damaging cellular membranes and components.

GSH, GST and SOD play a critical role in the protection of tissues from the deleterious effects of activated $\mathrm{AFB}_{1}$ (Larsson et al., 1994). GSH is a tripeptide containing cysteine that has a reactive $-\mathrm{SH}$ group with reductive potency. It can act as a nonenzymic antioxidant by direct interaction of - $\mathrm{SH}$ group with ROS or it can be involved in the enzymatic detoxification of ROS, as a cofactor or a coenzyme (Janssen et al., 1993). GST catalyzes the conjugation of $\mathrm{AFB}_{1}-8,9$-epoxides with $\mathrm{GSH}$ to form $\mathrm{AFB}_{1}$ - epoxide- $\mathrm{GSH}$ conjugates thereby decreasing the intracellular glutathione content (Raney et al., 1992). On the other hand, SOD plays an important role in the elimination of ROS derived from the peroxidative process in liver tissues (Packer $\boldsymbol{e t}$ al., 1978). SOD removes superoxide by converting it to $\mathrm{H}_{2} \mathrm{O}_{2}$, which can be rapidly converted to water by catalase (Abdel-Wahhab et al., 2006, 2010). This observation supports our finding where we observed a significant dose- dependent decline in the levels of GSH, GST and SOD in AFs-treated rats. Such results were previously recorded by Saleh et al. (2007); Preetha et al., 2006) and Verma and Nair (2001).

In addition, oxidative stress may result in damage to critical cellular macromolecules including DNA, lipids and proteins (Breimer, 1990). Cellular fatty acids are readily oxidized by ROS to produce lipid peroxyl radicals which can subsequently propagate into MDA may result in the interaction with cellular DNA-MDA adducts (Shen et al., 1994, 1995). ROS that induced cellular damage were estimated by monitoring lipid peroxidation. This is a well-known indication of cellular damage caused by oxidative stress. In the current study, MDA showed a significant dose-dependent increase in AFS treated rats in dose and 
time dependent manner. These results supported the earlier findings that AFs-induced oxidative stress and increased lipid peroxidation (Abdel-Wahhab and Aly, 2003 and Abbès et al., 2008).

Generally the toxic effect produced by AFs on different parameters was explained by Towner et al. (2003) and Sohn et al. (2003) who suggest that AFs toxicity may ensue through the generation of ROS like superoxide anion, hydroxyl radical and hydrogen peroxide $\left(\mathrm{H}_{2} \mathrm{O}_{2}\right)$ during the metabolic processing of $\mathrm{AFB}_{1}$ by cytochrome $P 450$ in the liver. These species may attack soluble cell compounds as well as membranes, eventually leading to the impairment of cell functioning and cytolysis (Berg et al., 2004). In addition, $\mathrm{AFB}_{1}$ is converted to its epoxide form by mixed function oxygenases. $\mathrm{AFB}_{1}-8,9$-epoxide being an electrophile spontaneously interacts with cellular macromolecules like DNA (AbdelWahhab et al., 2010 and Nayak and Sashidhar, 2010). Reaction occurs with high region specificity at the $N^{7}$ position of guanine residues in DNA to form adduct (Iyer et al., 1994).

HA, class of compounds resulting from decomposition of organic matter, particularly plants are natural constituents of drinking water, soil and lignite. It inhibit bacterial and fungal growth, thus decrease levels of mycotoxins in feed. Stress management, immune system, anti-inflamatory activity, antiviral properties as well as prevention of intestinal diseases, mainly diarrhoea in humans and animals are described as its beneficial effect (Islam et al., 2005).

This study has shown that HA alone does not have a negative impact on biochemical and oxidative stress parameters moreover HA enhance GSH production. The results obtained by different researchers (Lange $\boldsymbol{e t}$ al., 1996; Kühnert $\boldsymbol{e t}$ al., 1989) indicated that HA is toxicologically not harmful after oral administration.

The current results revealed that co-treatment with HA and AFs resulted in significant improvement in all tested parameters as indicated by significant decrease of elevated liver enzymes, creatinine, urea, cholesterol, triglycerides and glucose, significant increase of reduced concentration of TP, albumin, GSH, GST and SOD and decline of elevated serum MDA. Furthermore, HA at high dose $(400 \mathrm{mg} / \mathrm{kg}$ b.w) restored ALT, GGT, TP, albumin, creatinine, urea, triglycerides, GSH, GST and SOD to normal in rats treated with low AFs dose (1mg/kg b.w) by the end of experiment.

The beneficial properties of HA was previously reported in several researches against many toxic agents and diseases, Zhang et al. (2011) checked the toxicity and bioavailability of the ionic liquids (ILs), 1-butyl-3-methylimidazolium chloride and 1-octyl-3methylimidazolium chloride, in the presence of the HA. And find that the HA reduces the bioavailability and toxicity of these two ILs and also HA increases the viability around 50\% of HepG2 cell line against the two ILs. Moreover, the death rate of the model fish Medaka decreased against the lethal dose of two ILs when the HA was present in the water. Zralý et al (2008) have demonstrated that the feeding of lead along with HA reduces the accumulation of lead in chicken organs such as bone, muscle, liver and kidney, compared to the chicken feed without HA. Similarly, Herzig et al (2007) states that accumulation of cadmium were reduced in the presence of HA by $39.6 \%$ in kidney, $34.2 \%$ in liver and $80.8 \%$ in muscle as compared to the chicken feed with $\mathrm{Cd}$ in the absence of HA. Osman et al., (2009) concluded that $\mathrm{HA}$ is a promising tool for controlling cadmium pollution in aquaculture at short and long term, whereas it improves hematological, physiological and immunological state of $\mathrm{O}$. niloticus, exposed to cadmium, especially when it used at a concentration of $30 \mathrm{mg} / \mathrm{L}$. Also, it significantly reduces $\mathrm{Cd}$ level in fish tissues including musculsture. 
Furthermore, toxins, pesticides, radioactive metals, petroleum products, polyaromatic hydro-carbons and heavy metals can be inactivated by HA (Perminova and Hatfield, 2005 and Yates and Wandruszka, 1999). Moreover, it reduces bioavailability of the hazardous matters (Perminova and Hatfield, 2005) and averts the formation of the mutagenic or carcinogenic compound and prevents DNA damage in the cell.

Similar to our result, feeding broilers AFs-contaminated diet was associated with significant decreases in serum albumin, total protein, uric acid, and cholesterol. Compared with the control, the naturally contaminated diet significantly increased the activities of GGT, AST, and LDH. HA showed protective effects on feed efficiency and against liver and bursa of Fabricius damage, as well as some of the serum enzyme activities and serum biochemical changes associated with AF toxicity. These results suggest that HA (from $0.2 \%$ to $0.4 \%$ ) might be sufficient to counteract the adverse effects of AF (Ghahri et al., 2009). Further Van Rensburg et al. (2006) demonstrated that oxihumate was able to effectively bind $\mathrm{AFB}_{1}$ in vitro and was also effective in diminishing the growth inhibitory effects of AFs in vivo moreover the liver damage, stomach and heart enlargement in boiler chicken which occur after intake of AFs were reduced in presence of oxihumate. In addition, the serum toxicity caused by the toxin was also found to be reduced significantly. In another study, Santosa et al (2011) checked the absorption of the AFs by the HA polymer in the digestive system (oral cavity, stomach and intestines) at different $\mathrm{pH}$ of monogastric animals. HA can inactivate the AFs produced by the fungi.

The protective effect of HA explained by the ability of HA to bind AFs (Van Rensburg et al. 2006). Due to the colloidal characteristics and the ability to form chelates, HA and their salts can significantly modify the toxic effects of a number of xenobiotics and undesirable substances that enter the digestive tract together with feeds and water (Livens, 1991). Moreover, HA compounds are excellent antioxidants (Van Rensburg and Naude, 2009 and Lown et al, 2006) along with anti-lipid peroxidative activity and have antiinflammatory properties (Van Rensburg and Naude, 2009).

In an experimental model with partially hepatectomised rats, long-term application of HA resulted in the stimulation of omithine decarboxylase, an increase in spermidine and histamine as well as DNA and RNA levels, and in overall liver mass (Maslinski et al., 1993). It is also clear that the humate plays a role in the liver function and protects somewhat from disease and/or disturbances (Lotosh, 1991).

In conclusion, the results of the current study indicated that humic acid has a high affinity for aflatoxins in vitro and forms a stable adsorption complex. Humic acid is non toxic and effective to reduce the bioavailability of AFs in vivo and in the gastrointestinal tract, and prevents its biochemical toxicity. Humic acid can be used as sponge sequestering AFs in the gastrointestinal tract of farm animals.

\section{REFERENCES}

Abbès, S.; Abbès, J.B-S.; Hetta, M.M.; Ibrahim, M.; Abdel-Wahhab, M.A.; Bacha, H. and Oueslati, R. (2008): Efficacy of Tunisian montmorillonite for in vitro aflatoxin binding and in vivo amelioration of physiological alterations. Appl. Clay Sci., 42: $151-157$.

Abd El-Mageed, F.A. (1987): Some biological and nutritional studies on aflatoxins. M.Sc. Thesis, Zagazig Univ., Fac. of Agric., Egypt.

Abdelhamid, A.M.; Arief, H.S.M.; Elkeraby, F. and Dorra, T.M. (1995): Effect of some dietary supplements to aflatoxic diets of chickin II- effect on the tissue analysis. J. Agric. Sci. Mansoura Univ., 20 (7): 3227-3250. 
Abdelhamid, A.M. and Dorra, T.A. (1990): Study on the effect of feeding laying hens on separate mycotoxins (aflatoxin, patulin, or citrinin) contaminated diets on the egg quality and tissue contituents. Arch. Anim. Nut., Berlin. 40 (4): 305-315.

Abdel-Wahhab, M.A.; Ahmed, H.H. and Hagazi, M.M. (2006): Prevention of aflatoxin B1initiated hepatotoxicity in rat by marine algae extracts. J. Appl. Toxicol. 26 (3): 229-238.

Abdel-Wahhab, M.A. and Aly, S.E. (2003): Antioxidants and radical scavenging properties of vegetable extracts in rats fed aflatoxin-contaminated diet. J. Agric. Food Chem., 51 (8): 2409-2414.

Abdel-Wahhab, M.A.; Hassan, N.S.; El-Kady, A.A.; Khadrawy, Y.A.; El-Nekeety, A.A.; Mohamed, S.R.; Sharaf, H.A. and Mannaa, F.A. (2010): Red ginseng extract protects against aflatoxin B1 and fumonisins-induced hepatic pre-cancerous lesions in rats. Food Chem. Toxicol., 48 (2): 733-742.

Abdel-Wahhab, M.A. and Kholif, A.M. (2008): Mycotoxin in animal feeds and prevention strategies: a review. Asian J. Anim. Sci. , 2 (1): 7-25.

Abdel-Wahhab, M.A.; Nada S.A. and Khalil, F.A. (2002): Physiological and toxicological responses in rats fed aflatoxin-contaminated diet with or without sorbent materials. Animal feed Sci. Technol., 97: 209-219.

Abdel-Wahhab, M.A.; Omara, E.A.; Abdel-Galil, M.M.; Hassan, N.S.; Nada, S.A.; Saeed, A. and El-Sayed, M.M. (2007): Zizyphus Spina-Christi extract protects against aflatoxin $\mathrm{B}_{1}$-inhibited hepatic carcinogenicity. Afr. J. Trad. CAM 4 (3), 248256.

Alvarez-Puebla, R.A.; Goulet, P.J.G. and Garrido, J.J. (2005): Characterization of the porous structure of different humic fractions. Colloids Surf A Physicochem Eng. Asp., 256: 129-135.

Ankrah, N.A. (1995): Alteration of glucose tolerance in mice fed low levels of aflatoxins and with depressed glyoxalase-I activity. Vet. Hum. Toxicol., 37(1):59-61.

Autrup, J.L.; Schmidt, J.; Seremet, T. and Autrup, H. (1991): Determination of exposure to aflatoxins among Danish workers in animal feed production though the analysis of aflatoxin $\mathrm{B}_{1}$ adducts to serum albumin. Scand $\mathrm{J}$ Work Environ. Health, 17: 436-440.

Banu, G.S.; Kumar, G. and Murugesan, A.G. (2009): Ethanolic leaves extract of Trianthema Portulacastrum $L$. ameliorates aflatoxin $\mathrm{B}_{1}$ induced hepatic damage in rats. Indian J. Clinical Biochem., 24 (3): 250-256.

Bennett, J.W. and Klich, M. (2003): Mycotoxins. Clin. Microbiol. Rev., 16: 497-516.

Berg, D.; Youdim, M.B. and Riederer, P. (2004): Redox imbalance. Cell Tissue Res. 318, 201-213.

Beutler, E.; Duron, O. and Kelly, B.M. (1963): Improved method for the determination of blood glutathione. J. Lab Clin. Med.,61:882-888.

Breimer, L.H. (1990): Molecular mechanisms of oxygen radical carcinogenesis and mutagenesis: The role of DNA base damage. Mol Carcinogenesis, 3: 188-197.

Bullerman, L.B. (1986): Mycotoxins and food safety. Food Technol., 40: 59-66.

Choi, K.C.; Chung, W.T.; Kwon, J.K.; Yu, J.Y.; Jang, Y.S.; Park, S.M.; Lee, S.Y. and Lee, J.C. (2010): Inhibitory effects of quercetin on aflatoxin $\mathrm{B}_{1^{-}}$induced hepatic damage in mice. Food Chem. Toxicol., 48: 2747-2753. 
Choi, Y.K.; Jung, K.K.; Chae, K.Y.; Jang, I.; Lee, B.D.; Cole, R.A. and Cox, RH. (1981): Handbook of Toxic Fungal Metabolites. New York: Academic Press. Inc.,1.

Cozzi, R.; Nicolai, M.; Perticone, P.; De Salvia, R., and Spuntarelli F. (1993): Desmutagenic activity of natural humic acids: Inhibition of mitomycin $\mathrm{C}$ and maleic hydrazide mutagenicity. Mutat. Res., 299:37-44.

Davis, N.D.; Dioner, V.L. and El-Dridye, D.W. (1966): Production of aflatoxins $\mathrm{B}_{1}$ and $\mathrm{G}_{1}$ by Aspergillus flavus in a semisynthetic medium. Appl. Microbiol., 14: $378-$ 380.

Doyle, M.P.; Applebaum R.S.; Brackett, R.E. and Marth, E.H. (1982): Physical, chemical and biological degradation of mycotoxins in foods and agricultural commodities. J. Food Prot., 45: 946-971.

Ebadollahi-Natanzi, A.; Ghahremani, M.H.; Monsef-Esfahani, H.R.; Minaei, M.B.; Nazarian, H. and Sabzevari, O. (2010): Evaluation of Antihepatotoxic Effect of Watercress Extract and its Fractions in Rats. Int. J. Pharmacol., 6:896-902.

Edrington, T.S.; Kamps-Holtzapple, C.A.; Harvey, R.B.; Kubena, L.F.; Elissalde, M.H. and Rottinghaus, G.E. (1995): Acute hepatic and renal toxicity in lambs dosed with fumonisin containing culture materials. J. Anim. Sci. 73, 508-515.

Elfarissi, F., and Pefferkorn, E. (2000): Kaolinite/humic acid interaction in the presence of aluminium ion. Colloids Surf., A 168:1-12.

El-Nekeety, A.A.; Mohamed, S.R.; Hathout, A.S.; Hassan, N.S.; Aly, S.E. and AbdelWahhab, M.A. (2011): Antioxidant properties of Thymus vulgaris oil against aflatoxin-induce oxidative stress in male rats. Toxicon., 57: 984-991.

El-Zahar, H.; Tharwat, E.E.; Elaal, W.A.; El-Ashry, M.A.; Saad, M.M. and Amin, S.O. (1996): Rabbit and aflatoxins. 2- Reproductive performance of mature New Zealand white rabbit bucks treated orally with aflatoxins. Egyptian J. Rabbit Sci., 6(1): $67-78$.

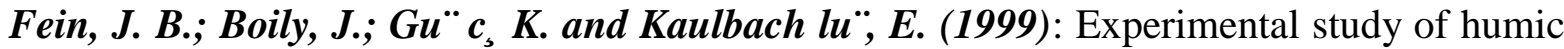
acid adsorption onto bacteria and Al-oxide mineral surfaces. Chem. Geol., 162:33-45.

Fink-Gremmels, J. (1999): Mycotoxins: their implications for human and animal health. Vet. Q., 21:115-120.

Ghahri, H.; Habibian, R. and Abdollah Fam, M. (2010): Evaluation of the efficacy of esterified glucomannan, sodium bentonite, and humic acid to ameliorate the toxic effects of aflatoxin in broilers. Turk. J. Vet. Anim. Sci., 34(4): 385-391.

Ghahri, H.; Talebi, A.; Chanmani, M.; Lotfollahian, H. and Afzali, N. (2009): Ameliorative effect of EGM, SB, and humic acid on humoral immunity of broilers during chronic aflatoxicosis. Turk. J. Vet. Anim. Sci., 33: 419-425.

Habig, W.H.; Pabst, M.J. and Jakoby, W.B. (1974): Glutathione S-transferases. The first enzymatic step in mercapturic acid formation. J. Biol. Chem., 25;249(22):71307139.

Hartenstein, R. (1981): Sludge decomposition and stabilization. Sci., 212:743-749.

Herzig, I.; Navratilova, M.; Suchy, P.; Vecerek, V. and Totus, J. (2007): Model trial investigating retention in selected tissues using broiler chicken fed cadmium and humic acid. Veterinarni Medicina., 52:162-168. 
Hsieh, D. (1988): Potential human health hazards of mycotoxins, p. 69-80. In S. Natori, K. Hashimoto, and Y. Ueno (ed.), Mycotoxins and phytotoxins. Third Joint Food and Agriculture Organization/W.H.O./United Nations E? Program International Conference of Mycotoxins. Elsevier, Amsterdam, The Netherlands.

Ioone, G.K.; Dekker, I. and Jansen van Rensburg, C.E. (2003): Investigation of the immunostimulatory properties ofoxihumate. Z Naturforsch C, 58:263-267.

Islam, K.M.S.; Schuhmacher, A. and Gropp, J.M. (2005): Humic acid substances in animal agriculture. Pakist. J Nutr., 4:126-134.

Iyer, R.S.; Coles, B.F.; Raney, K.D.; Their, R.; Lee Guengerich, F.P. and Harris, T.M. (1994): DNA adduction by the potent carcinogen aflatoxin $B_{1}$ : Mechanistic studies. J. Am. Chem. Soc., 166(5): 1603-1609.

Janssen, Y.M.; Van Houten, B.; Borm, P.J. and Mossman, B.T. (1993): Cell and tissue responses to oxidative damage. Lab. Invest. 69: 261-274.

Kanchana, K.; Hedhayathullahkhan, H.B.; Vedagiri, A.; Palanivelu, S. and Panchanatham, S. (2013): Pharmacological effect of Kalpaamruthaa on renal and cardiac damage induced by ingestion of aflatoxin $\mathrm{B}_{1}$ studied in wistar rats. Biomedicine \& Preventive Nut., 3:233-240.

Keçeci, T.; Oðuz, H.; Kurtoglu, V. and Demet, Ö. (1998): Effects of polyvinyl polyprolidone, synthetic zeolite and bentonite on serum biochemical and haematological characters of broiler chickens during aflatoxicosis. Brit. Poult. Sci., 39: 452-458.

Kensler, T.W.; Roebuck, B.D.; Wogan, G.N. and Groopman, J.D. (2011): Aflatoxin: a 50year odyssey of mechanistic and translational toxicology. Toxicol. Sci., 120 (S1), S28-S48.

Klein, P.J.; Van Vleet, T.R.; Hall, J.O. and Coulombe Jr., R.A. (2002): Biochemical factors underlying the age-related sensitivity of turkeys to aflatoxin $\mathrm{B}_{1}$. Comp. Biochem. Physiol., C 132: 193.

Klocking, R. (1994): Humic substances as potential therapeutic. In: Senesi N, Miano TM (eds): Humic substances in the global enviromnent and implication for human health. Elsevier Science B. V., Amsterdam: 1368 p., ISBN 0-444-89593-0

Klocking, R.; Sprossig, M.; Witzler, P.; Thiel, K.O. and Helbig, B. (1983): Antiviral wirkssame buminsaureahnliche Polymere. Z Phywther, 33:95-101.

Kollist-Siigur, K., Nielsen, T. Grøn, C. Hansen, P. E. Helweg, C. Jonassen, K. E. Jørgensen, $O$. and Kirso, $U$. (2001): Sorpsion of polycyclic aromatic compounds to humic and fulvic acid HPLC column materials. J. Environ. Qual., 30:526-537.

Kubena, L.F; Harvey, R.B.; Huff, W.E.; Elissalde, M.H.; Yersin, A.G.; Phillips, T.D. and Rottinghaus, G.E. (1993): Efficacy of a hydrated sodium calcium aluminosilicate to reduce the toxicity of aflatoxin and diacetoxyscirpenol. Poult. Sci., 72: 51-59.

Kühnert, V.M.; Fuchs, V. and Golbs, S. (1989): Pharmakologish-toxikologische Eigenshaften von Huminsäuren und ihre Wirkungsprofile für eine veterinarmedizinische Therapie. Deutsche Tierärztl. Wschr, 96: 3-10.

Kumar, R.; Mishra, A.K.; Dubey, N.K. and Tripathi, Y.B. (2007): Evaluation of Chenopodium ambrosioides oil as a potential source of antifungal, antiaflatoxigenic and antioxidant activity. Int. J. Food Microbiol., 115: 159. 
Lane-Peter, W. and Pearson, A.E.G. (1971): Dietary requirement cited in "The laboratory animal principles and practice". $1^{\text {st }}$ Academic press, London and New York. 142.

Lange, N.; Kühnert, M.; Haase, A.; Höke, H. and Seubert, B. (1996): Studies concerning the resorption properties of a low molecular humic substance after single oral application to rats. Dtsch. Tierärztl. Wschr, 103: 134-135.

Larsson, P.; Busk, L. and Tjalve, H. (1994): Hepatic and extrahepatic bioactivation and GSH conjugation of aflatoxin $B_{1}$ in sheep. Carcinogenesis 15, 947-955.

Levey, A.S.; Bosh, J.P.; Lewis, J.B.; Green, T.; Rogers, N. and Roth, D. (1999): A more accurate method to estimate glomerular filtration rate from serum crearinine: a new prediction equation. Modification of diet in renal disease study group. Ann. Int. Med. 130: 461-470.

Livens, F.R. (1991): Chemical-reactions of metals with humic material. Environ. Pollut., 70: 183-208.

Lotosh, T.D. (1991): Experimental bases and prospects for the use of humic acid preparations from peat in medicine and agricultural production. Nauchnye Doki Vyss Shkoly Biol. Nauki., 10: 99-103.

Lown, J.F.; Gill, K.; Cutler, S.J.; Cutler, H.G. and Pollock, S.H. (2006): Inventor; Antiinflammatory humate compositions and methods of use thereof. Dallas, TX (US).

MacCarthy, P. (2001): The principles of humic substances. Soil Sci., 166: 738-751.

Madronova', L.; Kozler, J.; Cezı'kova', J.; Nova'k, J. and Janoš, P. (2001): Humic acids from coal of the North-Bohemia coal field. III. Metal-binding properties of humic acids - measurements in a column arrangement. React. Func. Polym., 47:119-123.

Maslinski, C., Fogel, W. A. and Andrzejewski, W. (1993): An examination of humate stimulated liver functions. Acta Pol. Pharm., 50(4-5):413-416.

Meki, A. M. A.; Esmail, E.E.F.; Hussein, A.A. and Hassanein, H.M. (2004): Caspase- 3 and heat shock protein-70 in rat liver treated with aflatoxin $B_{1}$ : effect of melatonin. Toxicon, 43: 511-517.

Nanny, M. A., and Maza, J. P. (2001): Noncovalent interactions between monoaromatic compounds and dissolved humic acids: A deuterium NMR T1 relaxation study. Environ. Sci. Technol., 35:379-384.

Nayak, S. and Sashidhar, R.B. (2010): Metabolic intervention of aflatoxin $\mathrm{B}_{1}$ toxicity by curcumin. J. Ethnopharmacol, 127:641-644.

Ne'gre, M.; Schulten, H. R.; Gennari, M. and Vindrola. D. (2001): Interaction of imidazolinone herbicides with soil humic acids. Experimental results and molecular modeling. J. Environ. Sci. Health B, 36:107-125.

Nishikimi, M.; Rao, N.A. and Yagi, K. (1972): The occurrence of superoxide anion in the reaction of reduced phenazinemethosulfate and molecular oxygen. Biochem. Biophys. Res. Commun., 46: 849-854.

Onkawa, H.; Ohishi, N.; Yagi, K. (1979): Assay for lipid peroxides in animal tissues by thiobarbituric acid reaction. Anal. Biochem., 95:351-358.

Osman, H.A.M.; Taghreed, B.; Ibrahim, A.; Ali T. and Derwa, H.I.M. (2009): Field application of humic acid against the effect of cadmium pollution on cultured tilapia Oreochromis niloticus. World Appl. Sci. J., 6 (11): 1569-1575. 
Osuna, O. and Edds, G.T. (1982): Toxicology of aflatoxin $\mathrm{B}_{1}$, warfarin, and cadmium in young pigs: clinical chemistry and blood coagulation. Am .J. Vet. Res.,43: 387389.

Packer, J.E.; Slater, T.F. and Willson, R.L. (1978): Reactions of the carbon tetrachlonderelated peroxy free radical with amino acids: pulse radiolysis evidence. Life Sci., 23:2617-2620.

Perminova, I.V. and Hatfield, K. (2005): Remediation chemistry of humic substances: theory and implications for technology. In: Perminova IV, editor. Use of Humic substances to remediate polluted environments: From theory to practice. NetherlandS: Springer; p. 3-36.

Phillips, T.D.; Clement, B.A.; Kubena L.F. and Harvey, R.B. (1990): Detection and detoxification of aflatoxins: Prevention of aflatoxins and aflatoxin residues hydrated sodium calcium aluminosilicate. Vet. Human. Tox., 32: 15-19.

Pozzi, C.; Correa, B.; Xavier, J.; Direito, G.; Orsi, R. and Matarazzo, S. (2000): effects of prolonged oral administration of fumonisin $B_{1}$ and aflatoxin $B_{1}$ in rats. Mycopathologia, 151:21-27.

Preetha, S.P.; Kanniappan, M.; Selvakumar, E.; Nagaraj, M. and Varalakshmi, P. (2006): Lupeol ameliorates aflatoxin $\mathrm{B}_{1}$-induced peroxidative hepatic damagein rats. Comp. Biochem. Physiol., Part C Pharmacol. Toxicol., 143: 333-339.

Preston, R.J. and Williams, G.M. (2005): DNA-reactive carcinogens: mode of action and human cancer hazard. Crit. Rev. Toxicol., 35: 673.

Priegnitz, H. (1986): Wasserkur und Badelust. Leipzig: Koehler \& Amelang,S. 174-175.

Pumford, N.R.; Halmes, N.C. and Hinson, J.A. (1997): Covalent binding of xenobiotics to specific proteins in the liver. Drug Metab. Rev., 29:39-57.

Ramos, A. J. and Herna'ndez, E. (1996): In vitro aflatoxin adsorption by means of a montmorillonite silicate. A study of adsorption isotherms. Anim. Feed Sci. Technol., 62:263-269.

Ramos, A.J., Fink-Gremmels J. and Hernandez, E. (1996): Prevention of toxic effects of mycotoxins by means of nonnutritive adsorbent compounds. J. Food. Prot., 59: 631-641.

Raney, K.D.; Meyer, D.J.; Ketterer, B.; Harris, T.M. and Guengerich, F.P. (1992): Glutathione conjugation of aflatoxin $\mathrm{B}_{1}$ exo- and endo-epoxides by rat and human glutathione S-transferases. Chem. Res. Toxicol. 5, 470-478.

Rastogi, R.; Srivastava, A.K. and Rastogi, A.K. (2001): Long-term effect of aflatoxin $\mathrm{B}_{1}$ on lipid peroxidation in rat liver and kidney: Effect of picroliv and silymarin. Phytother.Res., 15(4):307-310.

Rath, N.C.; Huff, W.E. and Huff, G.R. (2006): Effects of humic acid on broiler chickens. Poult. Sci., 85: 410-414.

Roos, A. H.; Van der Kamp, H. J. and Marley, E. C. (1997): Comparison of immunoaffinity columns with Florisil/ C18 columns for the determination of aflatoxins in animal feed and maize. Mycotoxin Research, 13: 1-10.

Saini, S.S. and Kaur, A. (2012): Aflatoxin $\mathrm{B}_{1}$ : Toxicity, characteristics and analysis:Mini review. Global Advanced Res. J. of Chem. and Material Sci., 1(4): 63-70. 
Saleh, Z.A.; El-Garawany, G.A.; Assem, F. and El-Shibiny, S. (2007): Evaluation of the efficacy of whey protein to ameliorate the toxic effects of aflatoxins in rats. Inter. Dairy J., 17: 854-859.

Santosa, S.S.; Vermeulena, S.; Haritovab, A. and Fink-Gremmels, J. (2011): Isotherm modeling of organic activated bentonite and humic acid polymer used as mycotoxin adsorbents. Food Additives and Contaminants, 28(11):1578-1589.

Sato, T.; Ose, Y.; Nagase, H. and Hayase, K. (1987): Adsorption of mutagens by humic acid. Sci. Total Environ., 62:305-310.

Scheideler, S.E. (1993): Effects of various types of aluminosilicates and aflatoxin $\mathrm{B}_{1}$ on aflatoxin toxicity, chick performance, and mineral status. Pout. Sci., 72: 282-288.

Sharma, R.A. and Farmer, P.B. (2004): Biological relevance of adduct detection to the chemoprevention of cancer. Clin. Cancer Res., 10: 4901.

Shen, H.M.; Ong, C.H.; Lee, B.L. and Shi, C.Y. (1995): Aflatoxin B $\mathrm{B}_{1}$ induced 8hydroxydeoxyguanosine formation in rat hepatic DNA. Carcinogenesis, 16: 419422.

Shen, H.M.; Shi, C.Y.; Lee, H.P. and Ong, C.N. (1994): Aflatoxin $\mathrm{B}_{1}$ induced lipid peroxidation in rat liver. Toxicol. Appl. Pharmacol., 127: 145-150.

Sherif, S.O.; Salama, E. E. and Abdel-Wahhab, M.A. (2009): Mycotoxins and child health: the need for health risk assessment. Int. J. Hyg. Environ. Health, 212 (4): 347368 .

Sies, H. (1991): Oxidative Stress: Oxidants and Antioxidants. San Diego, California: Academic Press.

Snedecor, G.W. and Cochran, W.G. (1980): Statistical methods. $7^{\text {th }}$ (ed). Iowa state University Press, Ames, Iowa, USA.

Sohn, D.H.; Kim, Y.C.; Oh, S.H.; Park, E.J.; Li, X. and Lee, B.H. (2003): Hepatoprotective and free radical scavenging effects of Nelumbo nucifera. Phytomedicine, 10: 165-169.

Srivastava, A.k. (1984): Pharmaco-kinetics and therapeutic evaluation of oximes buffalo calves. Ph.D. Thesis ,Punjab Agriculture University, Ludhiana, India.

Stevenson, F.J. (1985): Geochemistry of soil humic substances. In: Aiken GR, McKnight DM, Wershaw RL, Maccarthy P, Eds. Humic Substances in Soil, Sediment and Water. New York: John Wiley \& Sons, pp13-52.

Terao, K. and Ueno, Y. (1978): Morphological and functional damage to cells and tissues. In: Toxicology, Biochemistry and Pathology of Mycotoxins, Uraguchi, K. and Adyamazaki, M. (eds.), Kodarsha Press, Tokyo, pp. 189-238.

Towner, R.A.; Qian, S.Y.; Kadiiska, M.B. and Mason, R.P. (2003): In vivo identification of aflatoxin-induced free radicals in rat bile. Free Radic. Biol. Med., 35: 13301340.

Van Rensburg, C.E.J. and Naude, P.J. (2009): Potassium humate inhibits complement activation and the production of inflammatory cytokines in vitro. Inflammation, 32(4):270-6.

Van Rensburg, C.J.; Van Rensburg, C.E.J.; Van Ryssen, J.B.J.; Casey, N.H. and Rottinghaus, G.E. (2006): In vitro and in vivo assessment of humic acid as an aflatoxin binder in broiler chickens. Poultry Sci., 85:1576-1583. 
Verma, R.J and Nair, A. (2001): Ameliorative effect of vitamin E on aflatoxin-induced lipid peroxidation in the testis of mice. Asian J Androl, 3: 217.

Verma, R.J. and Raval, P.J. (1992): Morphological alteration in rabbits under aflatoxininduced Toxaemia. J. Anim. Morphol. Physiol., 39: 95-98.

Wangikar, P.B.; Dwivedi, P.; Sinha, N.; Sharma, A.K.; Telang, A.G. (2005): Teratogenic effects inrabbits of simultaneous exposure to ochratoxin $A$ and aflatoxin $\mathrm{B}_{1}$ with specialreference to microscopic effects. Toxicol, 215:37-39.

Wogan, G.N. (1999): Aflatoxin as a human carcinogen. Hepatol., 30: 573-575.

Yates, L.M. and Wandruszka, R.V. (1999): Decontamination of polluted water by treatment with a crude humic acid blend. Environ. Sci. Technol., 33:2076-2080.

Ye, S.; Lv, X. and Zhou, A. (2009): In Vitro evaluation of the efficacy of sodium humate as an aflatoxin $B_{1}$ adsorbent. Aust. J. Basic \& Appl. Sci., 3(2): 1296-1300.

Zhang, Z.; Liu, J.; Cai, X.; Jiang, W.; Luo, W. and Jiang, G. (2011): Sorption to dissolved humic acid and its impacts on the toxicity of imidazolium based ionic liquids. Environ. Sci. Technol., 45:1688-1694.

Zralý, Z.; Písařiková, B.; Trčková, M. and Navrátilová, M. (2008): Effect of humic acids on lead accumulation in chicken organs and muscles. ACTA Vet. brno., 77:43945 .

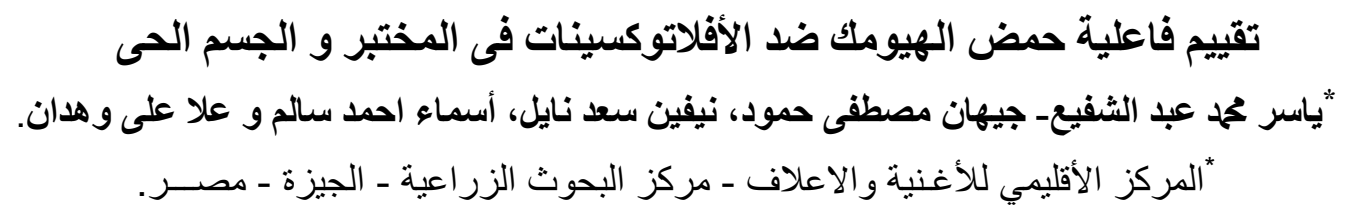

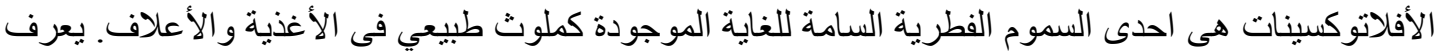

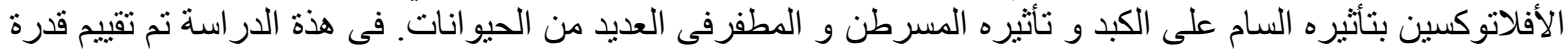

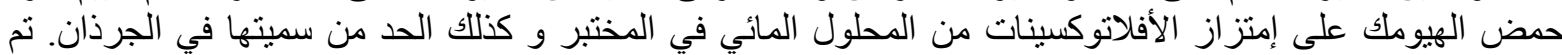

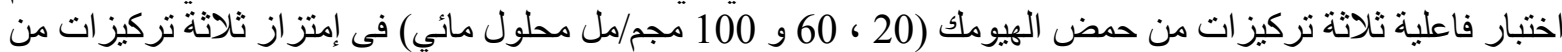

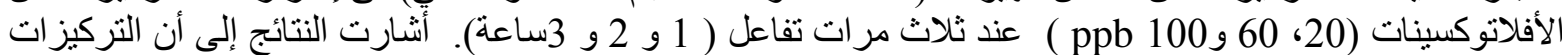

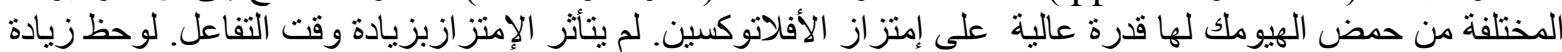

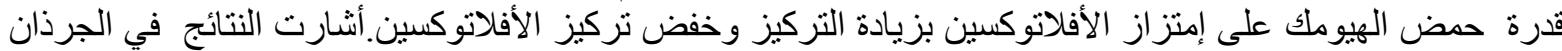

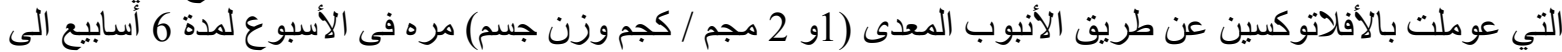

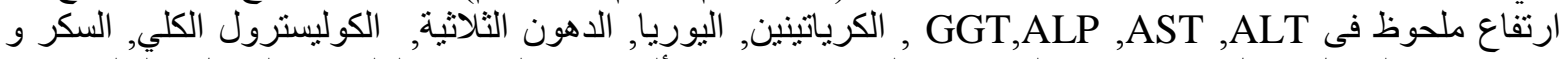

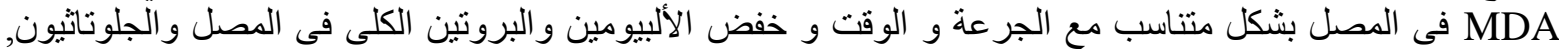

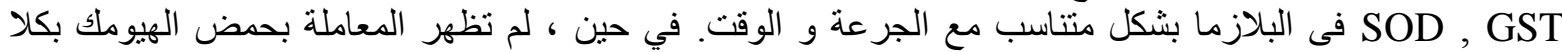

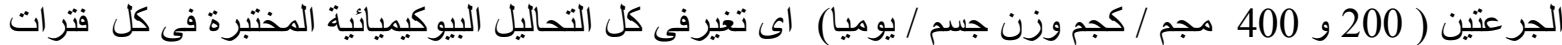

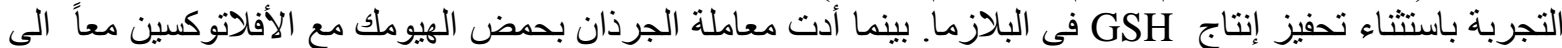

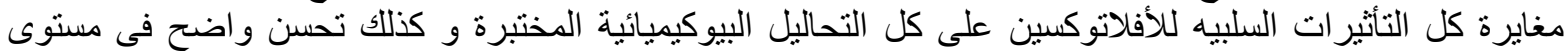

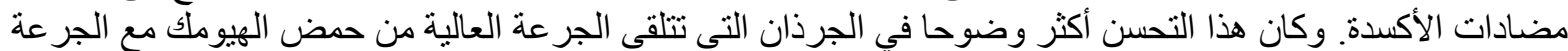

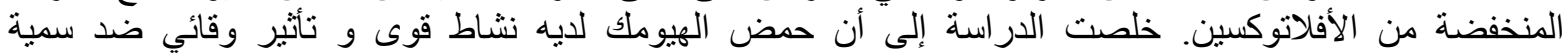

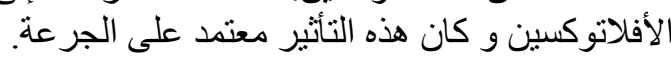
الكلمات الدالة: الأفلاتوكسينات, حضن الهين الهيومك, قدرة الإرتباط، الجهد المؤكسد و التحاليل البيوكيميائية. 